\title{
Antibody coated bacteria in urine of patients with recent spinal injury
}

\author{
A Galloway, H T Green, K K Menon, B P Gardner, S Pemberton, K R Krishnan
}

\begin{abstract}
Twenty patients with an acute spinal injury were prospectively studied to assess the clinical importance of antibody coated bacteria (ACB) in the urine and the association among the different bacterial species with a positive antibody coated bacteria test. Clinical urinary tract infection was associated with a positive ACB test on $45 \%$ of occasions. Three hundred and ninety nine urine samples containing 541 bacterial isolates were assessed for the presence of $\mathrm{ACB} ; 13 \%$ were found to be positive and $87 \%$ negative for $A C B ; 67 \%$ of urines contained a single bacterial isolate. Pseudomonas aeruginosa was most commonly associated with clinical urinary tract infection, found in $25 \%$ of episodes, followed by Proteus mirabilis (17.5\%), Klebsiella sp (12.5\%), and Proteus morganii (10\%). Providencia stuartii, however, was most commonly associated with a positive ACB test (found in 17\%). Other bacteria associated with a positive ACB test included Klebsiella sp (14\%), Acinetobacter sp (12.5\%), Pseudomonas aeruginosa (12\%), Citrobacter sp $(11 \cdot 5 \%)$.
\end{abstract}

A positive ACB test is not to be expected from a patient with spinal injury who has a catheter in place, and the test may provide a useful guide to identify those patients with an invasive infection. It is doubtful that a decision to treat or not treat bacteriuria could rest on the identification of the bacterial species alone.

\footnotetext{
Department of Microbiology, Southport General Infirmary, Merseyside A Galloway H T Green H T Green
$S$ Pemberton Regional Spinal Injuries Centre, Promenade Hospital, Southport, Merseyside K K Menon B P Gardner K R Krishnan Correspondence to: Dr A Galloway, Department of Microbiology, Dryburn Hospital, North Road, Durham, DH1 5TW

Accepted for publication 2 July 1990
}

urinary infections, such as prostatitis.

Thomas and Forland later reviewed the ACB test and concluded that the major clinical usefulness of a positive test was in distin- guishing a simple lower urinary tract infection from an invasive urinary tract infection which would require a prolonged course of antibiotics. ${ }^{2}$ The test has been used in patients with spinal injury with variable results. In 1980 Newman et al first described the use of the ACB test in patients with spinal injuryurine samples were obtained at the time of their annual review. ${ }^{3}$ They suggested that certain bacterial species were more likely than others to be antibody coated in the urine and would therefore warrant treatment with antibiotics. Conversely, it was suggested that infection with bacteria which were infrequently associated with a positive ACB test would be unlikely to warrant treatment in the absence of other signs or symptoms. We prospectively studied serial ACB tests in 20 patients with a recent spinal injury to assess the clinical importance of the ACB test and the association of the different bacterial species with the presence of ACB in the urine.

\section{Methods}

The patients comprised 17 males and three females, aged 12-67 years, admitted to the Regional Spinal Injuries Centre 0-38 days after injury (average seven days). Ten were tetraplegic and 10 were paraplegic. All required catheter drainage for at least 54 days and were studied for 14 days to 10 months (average six months). Daily clinical records were kept of any fever, signs or symptoms of sepsis, especially urinary tract infection, and antibiotic treatment.

Urine specimens and serum for C-reactive protein estimation were taken twice weekly, and when clinical urinary tract infection was suspected-for example, if the patient had rigors, a fever of more than $38^{\circ} \mathrm{C}$ for more than two hours, or positive blood cultures with significant bacteriuria $\left(>10^{5}\right.$ organisms/l of a catheter urine or $>10^{8}$ organisms $/ 1$ in a mid-stream sample) and pyuria ( $>10$ white cells $\left.\times 10^{6} / 1\right)$. Specimens were cultured aerobically overnight on cysteine lactose electrolyte deficient (CLED) agar. Those yielding significant bacteriuria were stored at $4^{\circ} \mathrm{C}$ for investigation of ACB in the urine. Cultures considered to be contaminated-that is two, or more bacterial species isolated from a midstream urine on a single occasion were excluded from the analysis.

\section{ACB TEST}

A modified immunofluorescent slide method developed by Matuscak et al was used. ${ }^{4}$ Urine 
Table 1 Bacterial species assocated with positive( +) and negative(-) ACB test organism isolated in pure culture

\begin{tabular}{lccc}
\hline Bacteria & ACB positive (\%) & ACB negative (\%) & Total \\
\hline Acinetobacter anitratus & $1(12 \cdot 5)$ & $7(87 \cdot 5)$ & 8 \\
Citrobacter sp & $3(11 \cdot 5)$ & $23(88 \cdot 5)$ & 26 \\
Enterobacter sp & $0(0)$ & $7(100)$ & 7 \\
Escherichia coli & $0(0)$ & $12(100)$ & 12 \\
Klebsiella sp & $3(14)$ & $19(86)$ & 22 \\
Proteus mirabilis & $5(11)$ & $42(89)$ & 47 \\
Proteus morganii & $0(0)$ & $1(100)$ & 1 \\
Providencia stuartii & $7(17)$ & $76(83)$ & 42 \\
Pseudomonas aeruginosa & $10(12)$ & $15(94)$ & 86 \\
Enterococcus faecalis & $1(6)$ & $2(100)$ & 16 \\
Coagulase negative staphylococci & $0(0)$ & Total & 2 \\
& & & 269 \\
\hline
\end{tabular}

$(0.5 \mathrm{ml})$ was centrifuged at $1500 \times g$ for 10 minutes. The supernatant was then discarded and the sediment was washed twice in phosphate buffered saline (PBS).

Smears of the sediment were prepared by placing one drop from a sterile 50 drop pipette into the well of the multispot slide and being air dried. Once dried, the smears were fixed in acetone at room temperature for 15 minutes. The slides were then stained by adding one drop of fluoroescein labelled antihuman immunoglobulin (Wellcome) to each spot and incubating in a moist chamber at $37^{\circ} \mathrm{C}$ for 30 minutes. Slides were washed by soaking in two changes of PBS for five minutes each. Finally, they were rinsed in distilled water and air dried. Slides were examined for fluorescence using a filter Y475. A semiquantative assessment was made of the number of ACB present and recorded as,,-+++ , or +++ . Control slides were prepared by suspending the different bacterial species isolated in PBS to just visible turbidity and then washing and staining them as above. Slides were examined for the presence or absence of fluorescence to exclude autofluorescence of any bacteria.

\section{Results}

Three hundred and ninety nine urine samples with positive culture were obtained during the study period and assessed for the presence of ACB, an average of 21 samples per patient (range five to 32). Thirteen percent of samples were positive and $87 \%$ were negative for ACB. The first urine sample positive for ACB after injury (and insertion of a catheter) was detected between five and 248 days after injury (mean 74 days) in 18 patients. During the study period 13 of the 20 patients developed 33 episodes of clinical urinary tract infection. In 32 of 33 episodes of clinical urinary tract infection

Table 2 Different bacterial species associated with clinical urinary tract infection

\begin{tabular}{lll}
\hline Bacterial isolate & Number isolated & Percentage of total \\
\hline Pseudomonas aeruginosa & 10 & 25 \\
Proteus mirabilis & 7 & 17.5 \\
Klebsiella sp & 5 & 12.5 \\
Proteus morganii & 4 & 10 \\
None & 4 & 10 \\
Citrobacter sp & 3 & 7.5 \\
Providencia stuartii & 3 & 7.5 \\
Enterococcus faecalis & 2 & 5 \\
Enterobacter sp & 1 & 2.5 \\
Escherichia coli & 1 & 2.5 \\
Total & 40 & \\
\hline
\end{tabular}

serum C-reactive protein was increased. In the one episode of clinical urinary tract infection when C-reactive protein was not increased, the ACB test was positive. All patients with clinical urinary tract infection received antibiotic treatment and the ACB test became negative in all cases after successful treatment for urinary tract infection, although in one patient the test remained positive for three weeks after a course of appropriate antibiotics had been started. On 19 occasions a positive ACB test was found when clinical urinary tract infection was not suspected. On five of these occasions an increased serum C-reactive protein concentration was also noted, raising the suspicion of a subclinical urinary tract infection, but the ACB test result became negative without specific treatment as antibiotic treatment was only given to those patients with clinical urinary tract infection.

Two tetraplegic patients who were studied for 120 and 300 days, respectively, did not at any time develop a positive ACB test despite persistent bacteriuria. One of these patients did, however, have one episode of clinical urinary tract infection during the study. In the 11 patients in whom ACB were present within two months of injury 10 were clinically known or suspected of having a urinary tract infection at the time. One patient developed a weakly positive ACB test at day 20 but was not suspected of having a urinary tract infection, and the ACB test was subsequently negative without antibiotic treatment.

ACB in the urine for the first time preceded the onset of clinical pyelonephritis. Four patients showed no signs or symptoms of urinary tract infection during the study, but three of these had ACB in the urine on at least one occasion, and in one patient this was associated with an increased C-reactive protein concentration on six separate occasions. Three other patients had a mild fever of less than $38^{\circ} \mathrm{C}$ with a positive urine culture and no other sites of sepsis (possible urinary tract infection). All had raised C-reactive protein concentrations and two out of three had ACB in the urine.

During the study 399 positive urine samples were obtained and 541 bacteria were isolated. Pseudomonas aeruginosa was the most common isolate, being present in 130 samples $(24 \%)$. Providencia stuartii, Proteus mirabilis, Klebsiella sp were also frequently isolated in 96 $(18 \%), 71(13 \%)$, and $69(12 \cdot 6 \%)$ of urines, respectively. These common organisms were found in several different patients: Ps aeruginosa, $P$ stuartii, and $P$ mirabilis were isolated from 14 of 20 of the patients on at least two occasions and Klebsiella sp were similarly found in 13 of 20 patients. Enterococcus faecalis, Acinetobacter, and staphylococcal species were occasionally isolated in $6 \%, 5 \%$, and $<1 \%$ of urine samples, respectively.

Of the positive urine cultures, however, 269 $(67 \%)$ contained organisms in pure culture and $130(33 \%)$ were mixed cultures. Table 1 shows the different bacterial species isolated from urine samples containing pure cultures only. Again $P$ s aeruginosa was the commonest isolate found in 86 of $269(32 \%)$ urine samples. $P$ 
mirabilis and $P$ stuartii were isolated from $17 \%$ and $16 \%$ of urines, respectively. $P$ stuartii, however, was most commonly antibody coated, the ACB test being positive in $17 \%$ of urines containing this organism in pure culture. Klebsiella sp, $P$ s aeruginosa, Citrobacter $\mathrm{sp}$ and $P$ mirabilis were also common isolates which were antibody coated in $10-15 \%$ of urines from which they were isolated $(14 \%, 12 \%, 11.5 \%$, and $11 \%$, respectively). $A$ anitratus was infrequently isolated but found to be ACB positive on one occasion. In contrast, Enterobacter, $E$ coli, $P$ morganii and staphylococci were infrequently isolated and not found to be antibody coated when isolated in pure culture.

Seven per cent of the mixed urine cultures were positive for ACB and yielded 46 different isolates. $P$ s aeruginosa and $P$ stuartii were commonly associated with a positive ACB test. The clinical importance of a positive ACB test with mixed urine cultures, however, is difficult to assess.

If the different bacterial species associated with clinical urinary tract infection are assessed (table 2) the results indicate that $P$ s aeruginosa was most commonly found ( $25 \%$ ), followed by $P$ mirabilis (17.5\%), Klebsiella sp (12.5\%), and $P$ morganii $(10 \%)$. P stuartii and Citrobacter was found less frequently $(7 \cdot 5 \%)$. In six of the episodes mixed bacterial cultures were obtained, and on four occasions no bacterial growth was obtained from the urine samples.

\section{Discussion}

Clinical symptoms and signs of urinary tract infection are notoriously unreliable for determining the site of urinary tract infection especially in the patient with spinal injury who depends on a catheter. The problems of diagnosing urinary tract infection in the patient with a spinal cord injury caused us to investigate the use of non-invasive techniques as a guide to invasive urinary tract infection in these patients because antibiotics should be reserved for those with an invasive infection. The presence of ACB in the urine had been interpreted by previous workers as an indication of infection in the kidney. ${ }^{15}$ This was later questioned when it was realised that antibody could be secreted at any level of the urinary tract when tissue invasion had occurred. $^{6}$ Our previous work indicated that C-reactive protein may be a useful guide in the management of urinary tract infection in patients with spinal injury. ${ }^{7}$ We have now studied the use of the ACB test in patients with a recent spinal injury with a view to assessing the clinical importance of a positive test and identifying the different bacterial species associated with a positive test in patients who have not previously required the long term use of a catheter.

Direct tests for localisation of urinary tract infection, such as ureteric catheterisation ${ }^{8}$ and the bladder washout test ${ }^{9}$ are most reliable but uncomfortable, may even be hazardous, and are too time consuming to be adopted as a routine clinical procedure on a regular basis for several patients. In 1974 Thomas and co-workers developed the technique of detecting antibody coated bacteria in urine. ${ }^{1}$ Since then several studies have looked into the usefulness of the test for predicting the site of infection comparing the ACB test with direct methods. ${ }^{10}$ Mundt and Polk reviewed the use of the ACB test in 1979 and found an overall sensitivity of $83.1 \%$ and a specificity of $76.7 \% .^{11}$ The positive predictive value was $81.3 \%$ and the negative predictive value $78.8 \%$. Non-standardisation of the ACB test, however, produced a range of results from different workers. Gargan et al attempted to standardise the ACB test. ${ }^{12}$ They found that patients with urinary tract infection and low counts of ACB-that is $<1 \%$-responded as well as those with a negative ACB to treatment for urinary tract infection. The presence of more than $1 \%$ ACB in a midstream urine specimen identified those patients who were at a high risk of treatment failurethat is, those who had "more than a simple lower tract infection".

The ACB test has been studied in several different groups of patients-those with simple or recurrent urinary tract infection, ${ }^{1512}$ spinal injury and neurogenic bladder, ${ }^{313-17}$ and in children. ${ }^{18}$ Diverse opinions have emerged regarding the usefulness of the test in each group. Studies on patients with neurogenic bladder have produced conflicting results. Some workers indicated that the limitations of the test precluded its use. ${ }^{1315}{ }^{17}$ Others felt that the test could be of some benefit while acknowledging its limitations. ${ }^{31416}$

Newman et al studied 121 urine samples from patients with spinal cord injury at the time of annual review. ${ }^{3}$ Sixty-six $(55 \%)$ of these had a positive ACB test (compared with $13 \%$ in our study). Of the positive culture results, $42 \%$ contained a single bacterial isolate (compared with $67 \%$ in our study). They found that $E$ coli was the commonest single isolate and antibody coated in $54 \%$ of urines. This was an uncommon isolate in our centre. In contrast, we found $P$ stuartii was a common isolate and the organism most commonly antibody coated. PS aeruginosa seemed to be a predominant organism in both centres. The discrepancy in results may have been due to the fact that patients in our study were those with a recent spinal injury, but in Newman's study the patients had more long standing injury. Despite the high percentage of positive tests Newman et al concluded that the ACB test is a useful diagnostic aid for this group of patients for whom more invasive methods would be inappropriate. ${ }^{3}$

Lindan studied 234 patients two months to 14 years after injury (average four years) and found that the patients' urine was positive for ACB in $34 \%$ of samples. ${ }^{16}$ They found that Klebsiella and Enterobacter were most commonly antibody coated and it was suggested that, "infection with these organisms should be treated promptly and vigorously". The study also indicated that Pseudomonas, Serratia, $P$ stuartii and Citrobacter were practically never antibody coated, and it concluded that isolation of these organisms in the urine did not necessarily warrant antibiotic treatment. Our results suggest that Pseudomonas, Providencia, and 
Citrobacter cannot be assumed to be harmless opportunist organisms as they were often associated with a positive ACB test and clinical urinary tract infection.

Our results indicate that although certain bacteria may be more commonly antibody coated than others, it is doubtful that a decision to treat or not to treat bacteriura in the patient with a spinal injury could rest on the identification of infecting bacterial species alone. Clinical signs and symptoms are also unreliable in predicting which patients require treatment. Most of our patients with clinical evidence of urinary tract infection had increased C-reactive protein concentrations, but only $45 \%$ had a positive ACB test result, indicating that there may be a group of patients who have a more severe invasive infection. Conversely, the presence of a positive ACB test in the absence of any clinical symptoms or signs and a normal C-reactive protein may be interpreted as a possible false positive result-by repeating the urine culture, ACB test, and estimating serum C-reactive protein concentration, it should be possible to clarify the meaning of the test. The lower incidence of positive ACB test results in the urines of our patients than in those reported in other studies indicates that perhaps the ACB test may be more useful in the management of patients with a recent spinal injury than in those who are being assessed at annual review. A combination of clinical assessment, the ACB test, and C-reactive protein estimation may provide a simple screening procedure to distinguish those patients who have bacteriuria alone from those who have an invasive infection.

The work was supported by a grant from the Regional Research Committee, Mersey Regional Health Authority.
1 Thomas VL, Shelokov A, Forland M. Antibody coated bacteria in the urine and site of urinary tract infection. $N$ Engl J Med 1974;290:588-90.

2 Thomas VI, Forland M. Antibody-coated bacteria in urinary tract infections. Kidney Int 1982;21:1-7.

3 Newman E, Price M, Ederer GM. Urinary tract infection in patients with spinal cord lesions: antibody-coated bacteria tests as a diagnostic aid. Arch Phys Med Rehabil 1980;61:406-9.

4 Matuscak RR, Pasculle AW, Barnhart L, Atchison RW, Yee RB. Slide method for detection of antibody coated bacteria in urine sediments. J Clin Microbiol 1980;12:761-3.

5 Jones SR, Smith JW, Sandford JP. Localisation of urinary tract infection by detection of antibody coated bacteria in urine sediment. N Engl J Med 1974;290:591-3.

6 Mengoli C, Arosio E, Bonato D, et al. Diagnostic value and biological significance of antibody coated bacteria in urine. J Clin Pathol 1980;33:183-7.

7 Galloway A, Green HT, Windsor JJ, Menon KK, Gardner BP, Krishnan KR. Serial concentrations of C-reactive protein as an indicator of urinary tract infection in patients with spinal injury. J Clin Pathol 1986;39:851-5.

8 Stamey TA, Govan DE, Palmer JM. The localisation and treatment of urinary tract infections: the role of bactericidal urine levels as opposed to serum levels. Medicine (Baltimore) 1965;44:1-36.

9 Fairley KF, Bond AG, Brown RB, Habersberger P. Simple test to determine the site of urinary tract infection. Lancet 1971;ii:615-8.

10 Giamarellou H. Antibody coated bacteria in urine. When, where and why? J Antimicrob Chemother 1984;13:95-9.

11 Mundt KA, Polk BF. Identification of site of urinary tract Mundt KA, Polk BF. Identification of site of urinary tract
infections by antibody coated bacteria assay. Lancet 1979;ii:1172-5.

12 Gargan RA, Brumfitt W, Hamilton-Miller JMT. Antibody coated bacteria in urine: criterion for a positive test and its value in defining a high risk of treatment failure. Lancet 1983;ii704-6.

13 Kuhlemeier KV, Lloyd LK, Stover SL. Failure of antibody coated bacteria and bladder washout tests to localize infection in spinal cord injury patients. $J$ Urol 1983;130:729-32.

14 Hooton TM, O'Shaughnessy EJ, Clowers D, Mack L, Cardenas DD, Stamm WE. Localisation of urinary tract infection in patients with spinal cord injury. $J$ Infect Dis infection in patients

15 Hulter HN, Borchardt KA, Mahood JA, Gelber RH, Kelso $\mathrm{CL}$. Localisation of catheter induced urinary tract infections: Interpretation of bladder washout and antibody coated bacteria tests. Nephron 1984;38:48-53.

16 Lindan R. The significance of antibody coated bacteria in neuropathic bladder urines. Paraplegia 1982;19:216-9.

17 Merrit JL, Keys TF. Limitations of the antibody coated bacteria test in patients with neurogenic bladders. JAMA 1982;247:1723-5.

18 Lorentz WB, Resnick MI. Comparison of urinary lactic dehydrogenase with antibody coated bacteria in the urine sediment as means of localizing the site of urinary tract infection. Pediatr 1979;64:672-7. 